\title{
Identification of Faulty BGA Solder Joints in X-Ray Images
}

\author{
Mohammad S. Laghari, Member, IACSIT and Qurban A. Memon
}

\begin{abstract}
The advantages of $\mathrm{X}$-ray inspection are extensive in scope due to the ability of $X$-rays to see through packages including heat sinks, welds, encapsulation, and metallic shielding to reveal obscured connections and identify potential quality non-destructive issues. Many assembly line manufacturers rely on real-time X-ray technology to evaluate solder joint integrity for BGAs and other leadless packages that cannot be inspected by conventional vision systems. X-ray inspection is particularly beneficial to applications that involve advanced surface-mount packaging technologies such as Ball Grid Array $(B G A)$ used for integrated circuits. This paper proposes a method that relies on computer vision and image processing techniques that inspects each ball image of a BGA chip and indicates the faulty balls for further assessment. The defective balls that are typically circular change into elongated or elliptical shape. Therefore, it exploits the use of the basic geometric distinction between a circle (perfect soldered ball) and an ellipse or any other shape (ball with reshaped solder joint). A contour analysis method is defined in this paper in terms of the angle changes that occur along the outline profile of a solder joint by moving three equal distant points located on the border. This angle change is plotted to detect the faulty ball joints.
\end{abstract}

Index Terms-BGA, shape analysis, solder joints, X-ray techniques.

\section{INTRODUCTION}

Computer vision is a process to locate and recognize objects in digital images. It involves techniques from image processing, pattern recognition and artificial intelligence. Computer vision has been used in diverse areas of applications. One such important area is in the field of on-line or off-line visual inspection systems of microscopic applications [1]-[3].

$\mathrm{X}$-ray inspection systems are widely used by electronics and SMT (surface-mount technology) manufacturers to control the quality of the hidden solder joints of BGA, wafer bonding defects, FCA (flip-chip assemblies), CSP (chip scale packages), QFN (quad flat no lead), and flip-chip components.

X-ray image processing, fault detection, and failure analysis tools provide the ability to quantify and fine-tune manufacturing processes, improve quality, yield, reduce scrap, and decrease warranty returns. X-ray examination has become an exceptional tool for process improvement, design evaluation, and quality assessment. To be brief, having X-ray inspection capability can positively affect the entire lifecycle

Manuscript received September 9, 2014; revised December 23, 2014

The authors are with the Department of Electrical Engineering, United Arab Emirates University, P. O. Box: 15551, Al Ain, United Arab Emirates (e-mail: mslaghari@uaeu.ac.ae,qurban.memon@uaeu.ac.ae). of a product [4].

$\mathrm{X}$-ray imaging is beneficial because it allows the inspection of component interiors and multiple layers of the board simultaneously. This viewing allows the detection of faults such as broken internal traces, voids in solder joints, and other flaws that would possibly go undetected by inspection schemes that use visible light or infrared light.

Although, visual inspection is monotonous and faults are difficult to detect using visible light however, it is important to note that the idea is not intended to substitute the human's function but to make it easier for the casual user to identify the suspect areas (solder joints) for further modification. It merely simplifies the visualization of the suspect balls and allows the user to inspect them individually.

BGA packages are used to permanently-mount devices such as microprocessors. One of the major causes of defective BGA chipsets is the improper solder joints between adjacent balls. This causes undesired short between the affected balls and renders the chip dysfunctional. Detecting such flaws are currently relied on a human visualization of an $\mathrm{X}$-ray image or tested electrically of the undermined chip. If the chip has several hundreds of balls and the solder joints are marginal, then it may be undetected by the casual observer. The weakness of manual inspection is the large amount of time required that results in the fatigue of the operator and decrease in the efficiency of work.

Electrical testing need probe holes on the chipboard reducing the advantage of component miniaturization. Such techniques will be unreliable in the future when chips will have even more leads and spaced even more closely. These methods also have the shortcoming that they do not provide any quantitative information about why joints failed [5].

Other methods and equipments applied for electronic failure inspections are: optical microscopy, cross sectioning, BGA DYE\&PRY test (destructive analysis method), shear test, scanning electron microscopy, X-ray fluorescent spectroscopy, de-capsulation, scanning acoustic microscopy, etc.

Typical solder joint failures detected by these techniques included with the X-ray are: wetting problem of cut surfaces, voiding, broken solder joint, shorts or bridging, insufficient through-hole solder joints, detection of de-elimination, missing part or connection points, misalignment, unacceptable size and shape variations in solder volume, poor co-planarity, etc. [6], [7].

Artificial neural network algorithms have also been applied in a variety of X-ray application domains. A QuickCog PC system is applied and adapted to advanced problems in electronics manufacturing. An automated X-ray image inspection system in electronics manufacturing can be 
performed with advanced sensor technology [8].

Computer vision and image processing techniques have been applied that detects each ball of the BGA chip and flags the suspect balls for further evaluation. Techniques such as Connected Component Analysis and K-L transformation were used to distinguish between geometric shapes of circle and ellipse [9].

The contour analysis method described in the paper is based on the perimeter data of the ball joint. It is defined in terms of the angle changes that occurs along the perimeter of a ball joint image by moving three adjacent equidistant points located on the border. This angle change is plotted on a graph and faulty ball joints are flagged when the plots exceed a threshold value.

\section{BACKGROUND EQUATIONS}

The main idea behind the presented algorithm centers on the fact that the perfect ball tends to be circular while faulty ones are somewhat elongated (elliptical shape). From basic algebra, in an $x-y$ Cartesian coordinate system, the circle with center coordinates $(a, b)$ and radius $r$ is the set of all points $(x$, y) such that:

$$
(x-a)^{2}+(y-b)^{2}=r^{2}
$$

This equation, also known as Equation of the Circle, follows from the Pythagorean theorem applied to any point on the circle: as shown in Fig. 1, the radius is the hypotenuse of a right-angled triangle whose other sides are of length $x-a$ and $y-b$. If the circle is centered at the origin ( 0 $0)$, then the equation simplifies:

$$
\begin{gathered}
(x-0)^{2}+(y-0)^{2}=r^{2} \\
x^{2}+y^{2}=r^{2}
\end{gathered}
$$

The equation can be written in parametric form using the trigonometric functions sine and cosine as:

$$
\begin{aligned}
& x=a \cos \theta, \\
& y=b \sin \theta
\end{aligned}
$$

Then by plotting $x$ and $y$ values in (4) and (5) for all angles of $\theta$ between 0 and $2 \pi$ results in an ellipse (e.g. at $\theta=0, x=$ $a, y=0$ and at $\theta=\pi / 2, y=\mathrm{b}, x=0)$.

Squaring both (4) and (5) give:

$$
\begin{aligned}
& x^{2}=a^{2} \cos ^{2} \theta, \\
& y^{2}=b^{2} \sin ^{2} \theta
\end{aligned}
$$

Dividing (6) and (7) by $a^{2}$ and $b^{2}$ respectively gives:

$$
\frac{x^{2}}{a^{2}}=\cos ^{2} \theta,
$$

$$
\frac{y^{2}}{b^{2}}=\sin ^{2} \theta
$$

Adding (8) and (9) together gives:

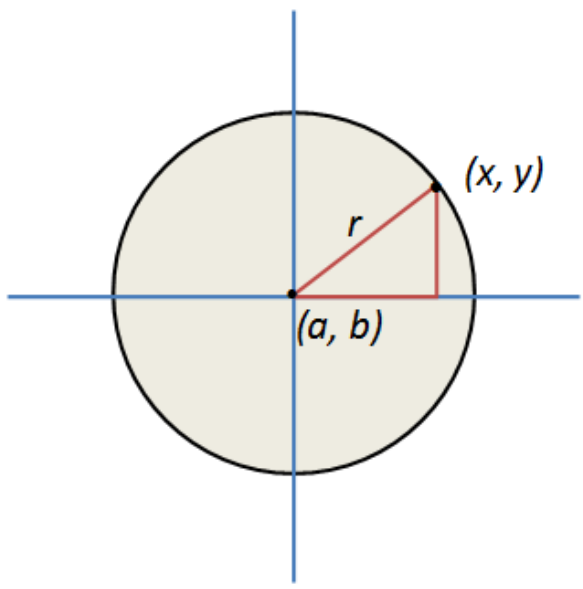

Fig. 1. Defining circle in terms of its parameters.

$$
\frac{x^{2}}{a^{2}}+\frac{y^{2}}{b^{2}}=\cos ^{2} \theta+\sin ^{2} \theta
$$

Applying the Pythagorean identity to the right hand side of (10) gives:

$$
\frac{x^{2}}{a^{2}}+\frac{y^{2}}{b^{2}}=1
$$

This means any noncircular ellipse is a compressed (or stretched) circle. If a circle is treated as an ellipse, then the area of the ellipse would be, proportional to the length of either axis (i.e. doubling the length of an axis in a circular ellipse would create an ellipse with double the area of the original circle).

\section{EXPERIMENTAL PROCEDURE}

Gray scale image of a section of the BGA displaying the solder joints is shown in Fig. 2. Image segmentation is used to distinguish between solder joints and the background. Out of several methods of image segmentation, thresholding is used to remove the gray level trends in an image, to make the gray level regions discrete, and split into distinct parts.

A devised procedure is used to identify individual ball joints for analysis. In this procedure, the stored gray scale image is threshold by setting all the gray levels below a certain level to " 0 " or above a certain value to a " 1 " corresponding to a maximum brightness level. Thus, a binary image of all the solder joints in the field of view is created. Thresholding converts a 256 gray level image into a binary image as shown in Fig. 3. By physical viewing of the binary image, it indicates the ball joints (encircled) with near perfect soldering joints as shown in Fig. 4.

Simple preprocessing may also be needed such as filtering to remove any undesired objects such as traces, silkscreen, etc. Since there are many ball joint images and the idea is locate the faulty ones, therefore a labeling technique is used to identify individual joints for analysis. 


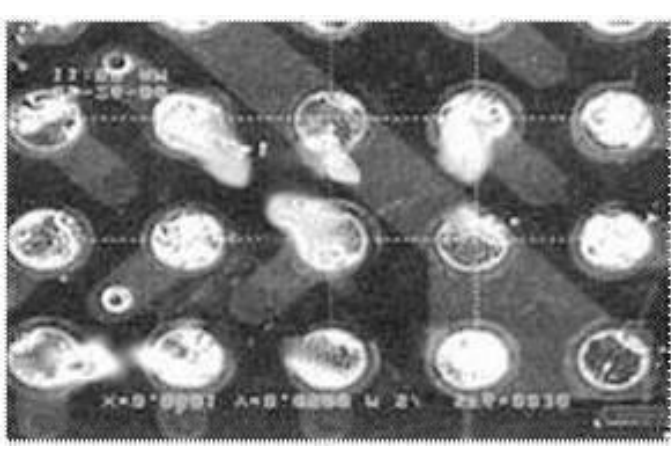

Fig. 2. A section of the BGA that shows some solder joints.

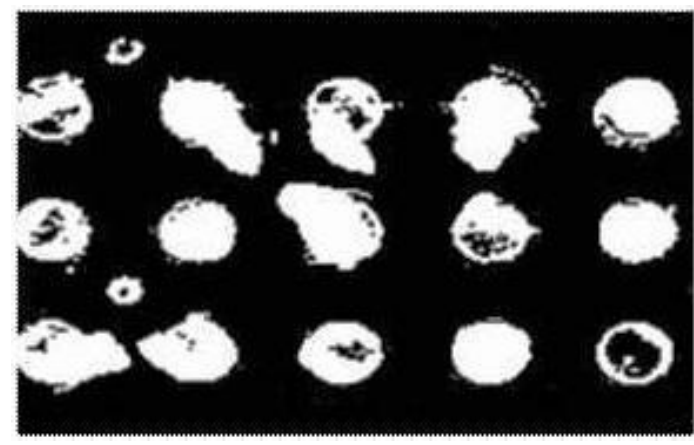

Fig. 3. Image after preprocessing and thresholding.

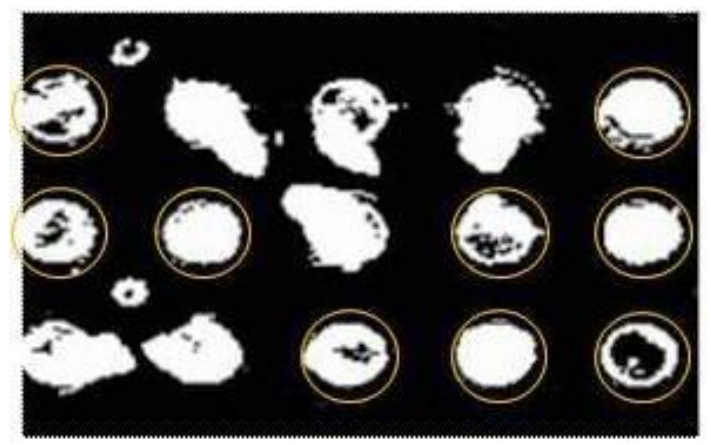

Fig. 4. Near perfect soldered ball joints are shown (encircled).

First, a neighboring connectivity scheme needs to be clarified. A ball joint pixel can have eight, four, or six neighbors. The way sample images are extracted; six-connectivity has been found suitable and used. There can be two six-neighbor-connectivity methods, which are (North West) NW/ SE (South East) or NE/SW.

The labeling process raster scan the image starting from right to left and label separate ball joints with different symbols. Therefore, by labeling individual soldering joints, an image filled with different numbers is created [10], [11].

The labeled soldering joints are area measured in terms of a pixel count. Most of the correct soldered joints have a near equal area measure with a narrow variation in the pixel count. All other image items such as chip mount, disjoint solders, etc., which are less than a specific surface area are eliminated. Fig. 5 shows the BGA image with eliminated unwanted elements.

The image is again raster scanned for every two contiguous horizontal lines at a time. Any change in intensity from background and labeled value or vice-versa horizontally or vertically between these two lines is recorded hence resulting in boundary detection operation of the ball joints image.

A boundary-tracking algorithm based on a chain code method is used which tracks the perimeter from pixel to pixel in a counter-clockwise starting from the right neighbor pixel of a selected wear particle and records its movement and stores all the points of the perimeter coordinates in the computer memory [12].

This perimeter data is subsequently then used to perform a contour analysis on every individual ball joint image. The contour analysis method is defined in terms of the angle changes that occur along the outline profile by moving three equal distant points located on the border. Two tangents are drawn through the points, which intercept one another to form an angle. The angle is measured from one tangent to another in such a way that it is positive when it rotates clockwise and negative when anti-clockwise rotation occurs, as shown from Fig. 6.

By plotting the measured angles around the complete ball joint boundary, it is possible to generate a contour pattern. Typical examples of contour patterns are shown in Fig. 7, Fig. 8, Fig. 9, and Fig. 10, respectively.

Fig. 7 shows a Radar chart of a near perfect solder joint whereas Fig. 8 is analyzed results of the reshaped ball joint and tends more to be an elliptical shape. One major and two minor variations in angle changes are shown with arrows in the figure.

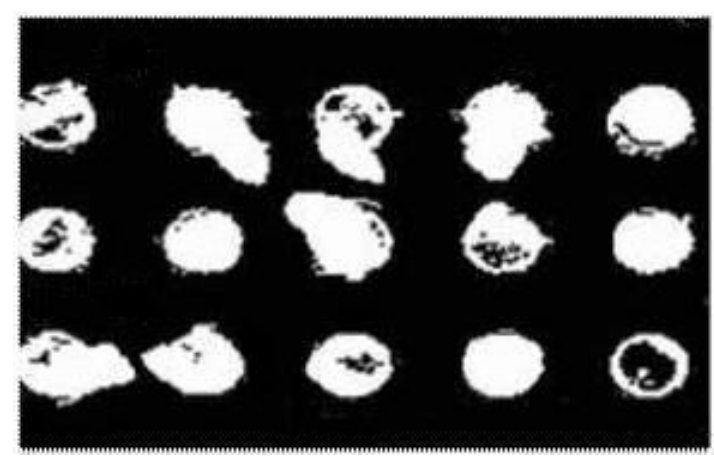

Fig. 5. Image with eliminated unwanted elements.

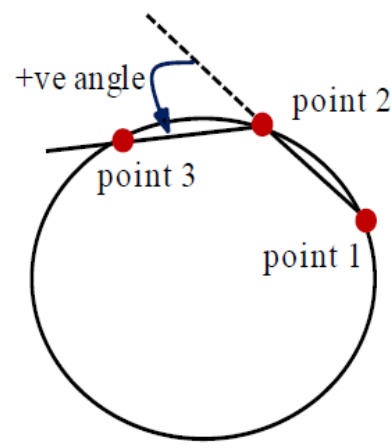

Fig. 6. The contour analysis with positive angle.

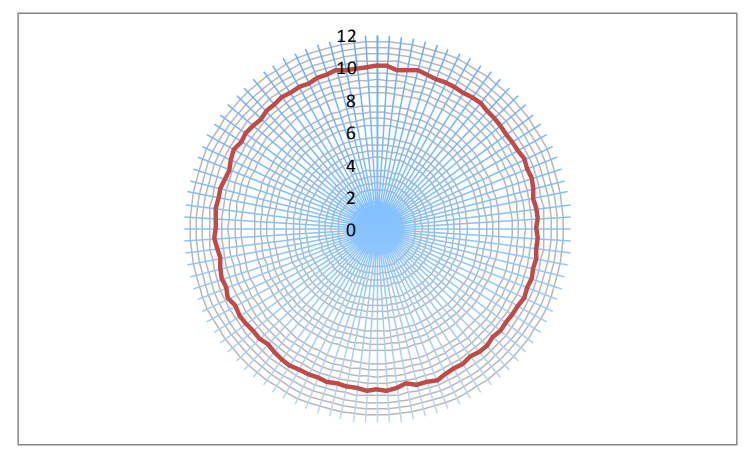

Fig. 7. Radar chart of a near perfect soldered ball joint. 


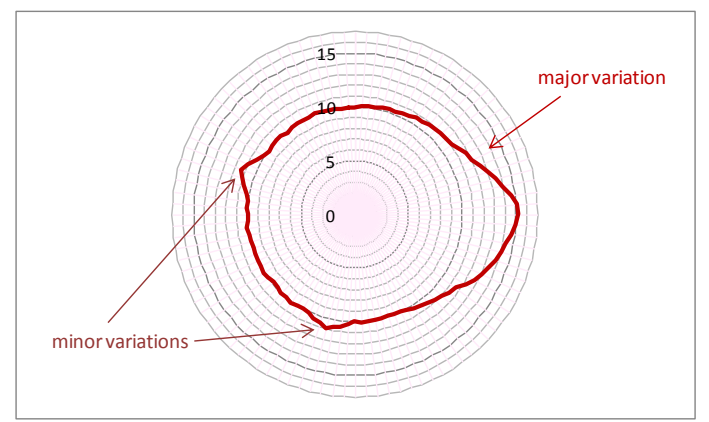

Fig. 8. Radar chart of a reshaped ball joint.

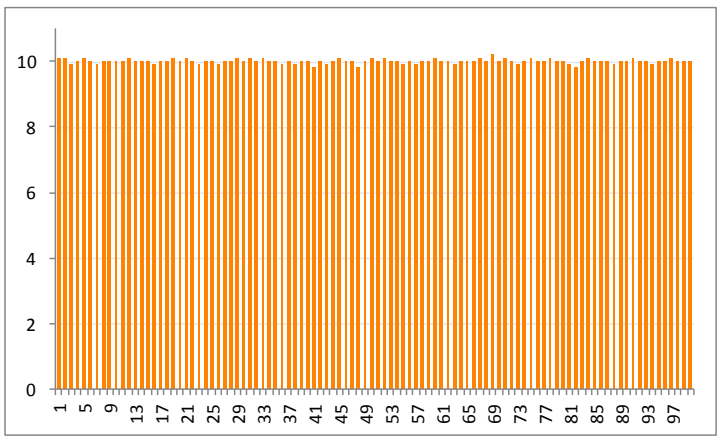

Fig. 9. Contour plot of the correctly soldered ball joint.

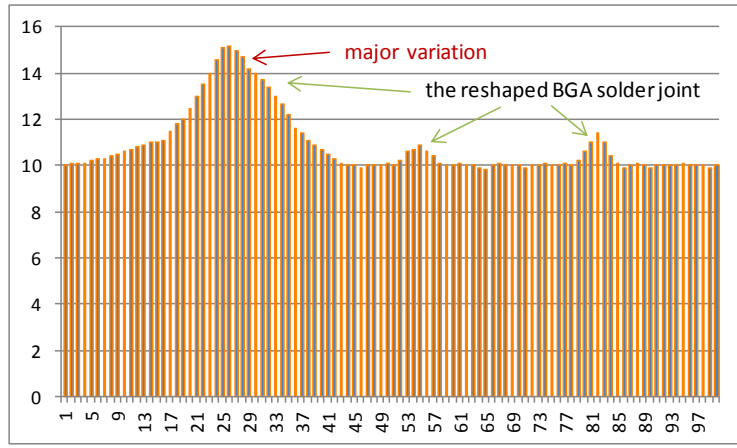

Fig. 10. Contour plot of the reshaped ball joint.

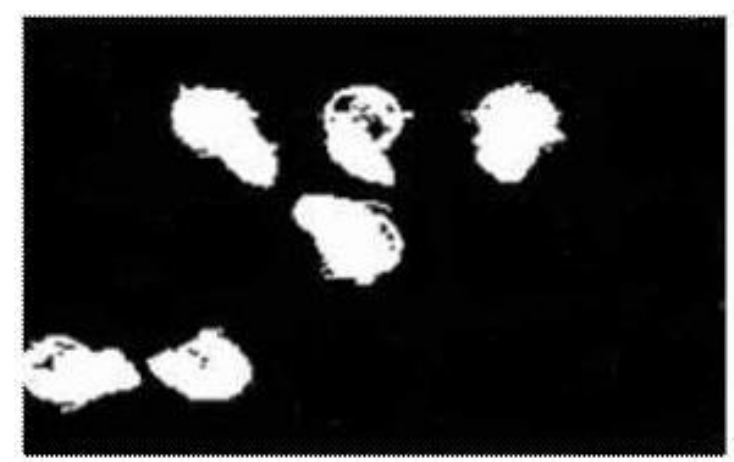

Fig. 11. BGA image showing only the faulty balls.

The positive activities of both of these examples are indicated in the XY charts as shown in Fig. 9 and 10, respectively.

The contour plot of the correctly soldered ball joint is indicated with most of the angle change activity is consistent except of the minor variations of the peak values. This is due to minor irregularities in the joint image, which is evident from Fig. 9.
The contour plot of the reshaped solder joint is shown in Fig. 10. The angle change activity is inconsistent as it moves along the joint contour. Any irregular shape of the ball joint is indicated with major and minor variations in the peak values as shown with arrows in the Figure. The minor variation is acceptable however the major variation is an indicative of a faulty soldered ball joint.

Fig. 11 shows the results with the correct solder joints filtered out.

\section{REFERENCES}

[1] M. S. Laghari and G. A. Khuwaja, "Scheduling techniques for parallel implementation of wear particle recognition algorithms," presented at the $3^{\text {rd }}$ International Conference on Digital Information Processing and Communications, Dubai, UAE, January 30-February 1, 2013.

[2] M. S. Laghari, F. Ahmed, and J. Aziz, "Wear particle shape \& edge detail analysis," presented at the $2^{\text {nd }}$ IEEE International Conference on Computer and Automation Engineering, Singapore, February 26-28, 2010.

[3] L. da F. Costa and R. M. Cesar Jr., Shape Classification and Analysis. Theory and Practice; Florida: CRC Press, 2009.

[4] B. L. Pierce, D. J. Shelton, H. G. Longbotham, S. Baddipudi, and P. Yan, "Automated inspection of through hole solder joints utilizing X-ray imaging," in Proc. the IEEE Systems Readiness Technology, Texas, Sep. 1993.

[5] E. Griffiths and R. Jordan, "Automatic inspection of surface mount solder joints using X-Ray images," in Proc. the BMVC, Oxford, 1990, pp. 157-162.

[6] B. Balogh, G. Harsanyi, R. Kovacs, C. Nagynemedi, and B. Papp, "Analysis of solder joint failures arisen during the soldering process," Periodica Polytechnica, vol. 52, no. 1-2, pp. 5-11, 2008.

[7] J. P. Hofmeister et al., "Ball grid array (BGA) solder joint intermittency detection: SJ BISTTM,” IEEEAC, vol. 1, Aug. 2007.

[8] A. König, A. Herenz, and K. Wolter, "Application of neural networks for automated X-ray image inspection in electronics manufacturing," LNCS, vol. 1607, pp. 588-585, 1999.

[9] M. S. Laghari, R. Hijer, and G. A. Khuwaja, "Efficient technique for BGA solder joints identification in low resolution X-ray images," presented at the $6^{\text {th }}$ IEEE GCC Conf. \& Exhibition 2011, Dubai, UAE, February 12-15, 2011.

[10] W. K. Pratt, Introduction to Digital Image Processing, Florida: CRC Press, 2013.

[11] D. G. Bailey and C. T. Johnston, "Single pass connected components analysis," in Proc. the Image and Vision Computing New Zealand IVCNZ, Hamilton, New Zealand, pp. 282-287, Dec. 2007.

[12] S. E. Umbaugh, Digital Image Processing and Analysis; Florida: CRC Press, 2011.

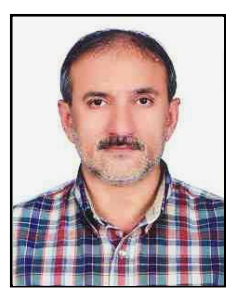

Mohammad S. Laghari was born in Karachi, Pakistan. He received his B.E. degree in electronic engineering from Mehran University of Engineering and Technology, Pakistan, M.S. degree in electrical engineering from Drexel University, Philadelphia, USA, and $\mathrm{Ph} . \mathrm{D}$. in computer engineering from the University of Wales, UK in 1980, 1983, and 1993, respectively.

He has taught at the Kuwait University, King Saud University and is currently an associate professor in the Electrical Engineering Department, United Arab Emirates University, U.A.E. His research interests are in applied artificial intelligence, image processing, pattern recognition, and software systems. He has published more than 70 papers in these areas.

Dr. Laghari is a member of the IACSIT (Singapore), PEC (Pakistan), UACEE (USA), and IRED (USA). 